\title{
Orientações para Assistência Nutricional a Pacientes com Câncer Avançado em Cuidados Paliativos Suspeitos ou Confirmados de Infecção pelo Novo Coronavírus
}

doi: https://doi.org/10.32635/2176-9745.RBC.2020v66nTemaAtual.1038

\author{
Orientations for Nutritional Assistance to Patients with Advanced Cancer in Palliative Care with Suspected or Confirmed New \\ Coronavirus Infection \\ Pautas para la Asistencia Nutricional a Pacientes con Cáncer Avanzado en Atención Paliativa Sospechada o Confirmada \\ de Nueva Infección con Coronavirus
}

Karla Santos da Costa Rosa'; Emanuelly Varea Maria Wiegert'²; Mariana Fernandes Costa; Rosane de Souza Santos ${ }^{4}$; Livia Costa de Oliveira $^{5}$

\section{INTRODUÇÃO}

A saúde pública mundial enfrenta atualmente um desafio: a pandemia originada pelo novo coronavírus, agente causador da doença denominada coronavirus disease 2019 (Covid-19), descoberta em 31 de dezembro de $2019^{1,2}$. O estado de pandemia, por sua vez, foi declarado em 11 de março de 2020 pela Organização Mundial da Saúde (OMS) ${ }^{1}$. Sabe-se que o vírus tem alta transmissibilidade e provoca a síndrome respiratória aguda grave do coronavírus 2 (severe acute respiratory syndrome coronavirus 2 - Sars-CoV-2), que varia de casos leves - cerca de $80 \%$ - a casos graves com insuficiência respiratória potencialmente letal - entre $5 \%$ e $10 \%$ dos $\operatorname{casos}^{2-4}$.

Pacientes com neoplasias malignas são mais suscetíveis a essa infecção quando comparados aos indivíduos saudáveis em razão do comprometimento do sistema imune, causado pela presença da doença e pelos tratamentos antitumorais ${ }^{5-7}$. No cenário dos cuidados paliativos oncológicos, esse grupo torna-se mais vulnerável à forma grave da Covid-19, atribuído ao maior risco de resultados desfavoráveis e ao pior prognóstico da doença avançada $^{7,8}$.

No contexto da pandemia, se faz fundamental a oferta de cuidados paliativos ${ }^{9}$ - abordagem voltada para melhoria da qualidade de vida de pacientes e familiares que enfrentam uma doença ameaçadora da vida, por meio da prevenção e alívio do sofrimento, da identificação precoce e tratamento da dor e de outros sintomas físicos, psicossociais e espirituais ${ }^{10}$.

No campo de conhecimento da nutrição e câncer avançado, náo foram encontrados estudos sobre o impacto da infecçáo pela Covid-19 no estado nutricional. Contudo, os achados que relacionam o estado nutricional com a infecçáo indicam que a desnutrição, a perda de massa muscular e a funcionalidade podem repercutir em piores resultados ${ }^{8,11}$. Quanto às alteraçôes laboratoriais, a anemia e a hipoproteinemia são frequentes em pacientes com neoplasias malignas, o que pode afetar a imunocompetência e aumentar a suscetibilidade ao Sars-CoV-2, sendo a hipoxemia e a dispneia sintomas comuns $^{8,11}$.

\section{ORIENTAÇÕES}

Diante da conjuntura da pandemia da Covid-19, tornou-se necessária a revisão das práticas, processos e protocolos assistenciais, visando à garantia da qualidade $\mathrm{e}$ da segurança dos pacientes e colaboradores que trabalham nas unidades de saúde do país ${ }^{12}$. Medidas de prevenção e controle de infecção no ambiente hospitalar devem ser implementadas para evitar ou reduzir ao máximo a transmissão e a disseminação do coronavírus durante qualquer assistência à saúde realizada ${ }^{13}$.

Como os pacientes com câncer em cuidados paliativos preenchem critérios de grupo de risco para a Covid-19, a Academia Nacional de Cuidados Paliativos (ANCP)

\footnotetext{
${ }^{1}$ Especialista. Nutricionista. Unidade de Cuidados Paliativos do Instituto Nacional de Câncer José Alencar Gomes da Silva (INCA). Rio de Janeiro (RJ), Brasil. Orcid iD: https://orcid.org/0000-0002-0951-8725

${ }^{2}$ Doutora. Nutricionista. Unidade de Cuidados Paliativos do INCA. Rio de Janeiro (RJ), Brasil. Orcid iD: https://orcid.org/00000001-5904-5287

${ }_{3}^{3}$ Doutora. Nutricionista. Unidade de Cuidados Paliativos do INCA. Rio de Janeiro (RJ), Brasil. Orcid iD: https://orcid.org/0000-0002-7702-1841

${ }^{4}$ Mestre. Nutricionista. Unidade de Cuidados Paliativos do INCA. Rio de Janeiro (RJ), Brasil. Orcid iD: https://orcid.org/00000002-9081-3501

${ }^{5}$ Doutora. Nutricionista. Unidade de Cuidados Paliativos do INCA. Rio de Janeiro (RJ), Brasil. Orcid iD: https://orcid.org/0000-0002-5052-1846

Endereço para correspondência: Karla Santos da Costa Rosa. Rua Visconde de Santa Isabel, 274, Vila Izabel. Rio de Janeiro (RJ), Brasil. CEP 20.560-120.

E-mail: kcostarosa@gmail.com
} 
orienta a suspensão dos atendimentos presenciais sempre que possível, com o objetivo de minimizar a exposição e a possível contaminação pelo vírus, priorizando, assim, o seguimento por teleconsulta nos atendimentos ambulatorial e domiciliar ${ }^{14}$.

$\mathrm{Na}$ modalidade de atendimento domiciliar, faz-se necessário reorganizar o fluxo de funcionamento e atendimento, seguindo as recomendaçôes publicadas pela OMS para assistir a pacientes suspeitos ou confirmados com a Covid-19, bem como seus contactantes ${ }^{15}$. Em virtude da maior exposição dos pacientes e dos profissionais de saúde durante as visitas domiciliares, recomenda-se sua redução, sempre que viável. Desse modo, tal como no atendimento ambulatorial, cada paciente deve ser avaliado quanto à necessidade de atendimento presencial, substituindo-o pela teleconsulta.

Caso a equipe interdisciplinar considere a necessidade de atendimento nutricional, este poderá ser realizado por meio teleconsulta, amparado pela Resolução do Conselho Federal de Nutricionistas (CFN) nº. 646, de 18 de março de 2020, que autoriza, em caráter de exceçáo, a assistência nutricional não presencial ${ }^{16}$.

\section{Pronto ATENDIMENTO}

Durante a pandemia da Covid-19, é recomendado cumprir o fluxo de planejamento emergencial desenvolvido por cada unidade hospitalar. O fluxo deverá ser específico por área assistencial, no qual pacientes admitidos com suspeita de contaminação pela Covid-19 deverão ser encaminhados para atendimento em local exclusivo para este fim ${ }^{13}$. Nos serviços de pronto atendimento, o nutricionista deverá planejar o cuidado nutricional a partir da avaliação realizada pela equipe médica e de enfermagem e mediante informaçóes obtidas por meio de fontes secundárias (familiares, prontuários ou outros integrantes da equipe interdisciplinar $)^{12}$.

\section{ATENDIMENTO AMBULATORIAL}

$\mathrm{O}$ atendimento presencial deve ser avaliado com cautela e pode ser ofertado conforme a necessidade do paciente e de acordo com as demandas a serem priorizadas pela equipe interdisciplinar e pactuadas com a gestão da organização institucional ${ }^{14,17}$.

Devem-se considerar as recomendaçóes de cada instituiçáo para o uso de equipamentos de proteçáo individual (EPI) e biossegurança, adequados para a assistência presencial ${ }^{13}$.

$\mathrm{O}$ uso de recursos físicos para avaliação nutricional nos casos não suspeitos, como fitas métricas, balanças, dinamômetros, adipômetros, entre outros, deve ser avaliado criteriosamente e, se utilizados, estes devem ser devidamente sanitizados com álcool a $70 \%$ entre cada atendimento ${ }^{12}$.
No suporte não presencial, devem-se considerar os dados secundários dos prontuários e empregar instrumentos subjetivos de triagem e avaliação nutricional, assim como parâmetros laboratoriais, avaliação dos sintomas e indicadores prognósticos que atendam às necessidades de cada serviço, os quais nortearão a assistência nutricional ${ }^{11,12,18,19}$.

Durante $\mathrm{o}$ atendimento, deve-se atentar para as queixas atuais do paciente e de seus familiares, correlacionando-as às suas condiçóes clínicas, laboratoriais e nutricionais, visando a um planejamento nutricional individualizado e que evite deslocamentos desnecessários ao serviço de saúde $e^{14,19}$.

Em decorrência da indicação de atendimento presencial com a necessidade de equipe interdisciplinar, recomenda-se que seja feito por um especialista por vez no local onde o paciente se encontrar, seguindo as precauçóes recomendadas ${ }^{13,14}$.

As orientaçóes fornecidas e o plano de cuidados nutricional devem ser registrados em prontuário ${ }^{19} \mathrm{e}$ compartilhados com a equipe assistente.

Ressalta-se, ainda, considerar seguimento por teleconsulta e a possibilidade de prolongamento do intervalo entre as consultas subsequentes, conforme anamnese nutricional, quadro clínico, controle de sintomas e prognóstico do paciente e/ou de acordo com as suas necessidades emergentes ou de seus familiares.

\section{ATENDIMENTO DOMICILIAR}

No atendimento domiciliar presencial ou por meio teleconsulta, quando houver a identificação de demandas nutricionais, a equipe interdisciplinar deve acionar o nutricionista referenciado para atendimento.

Orientaçōes nutricionais devem, preferencialmente, ser fornecidas de forma remota pelo nutricionista aos outros profissionais da equipe em contato com o paciente e familiares ou cuidadores, reduzindo assim a necessidade de agendamento de consultas para atendimento presencial $^{14,17}$. O planejamento nutricional deve ser registrado em prontuário ${ }^{19} \mathrm{ou}$ em formulário próprio para acesso de toda equipe ${ }^{17}$.

O nutricionista deve promover, sempre que possível, educaçáo alimentar e nutricional ao paciente e a seu núcleo de cuidados. As demandas de atendimento nutricional deverão ser priorizadas, entre as quais, estão as orientaçôes nutricionais para controle de sintomas de impacto nutricional, como náuseas, vômitos, hiporexia, disfagia, constipação, mucosite, entre outros, e prescrição nutricional de dieta enteral, caso a via alimentar tenha sido implementada recentemente ou pela primeira vez ${ }^{19}$. Devem-se fornecer orientaçóes sobre conteúdo, preparo e a correta manipulação e administração da dieta; além da 
readequação da conduta nutricional em razão de possíveis intercorrências relacionadas à terapia nutricional, como intolerância ao volume prescrito, diarreia, constipação, distensão abdominal, entre outras.

\section{INTERNAÇÃO HOSPITALAR}

O nutricionista que atua em ambiente hospitalar deve adotar medidas de higiene pessoal e uso de EPI adequados durante toda a permanência na unidade hospitalar, seguindo rigorosamente as orientaçôes da Comissão de Controle de Infecção Hospitalar de sua instituiçãao ${ }^{12,13,17}$.

Todos os pacientes com câncer avançado em cuidados paliativos internados com suspeita de Covid-19 ou confirmação do diagnóstico devem receber assistência nutricional ${ }^{12,18}$. Os objetivos da abordagem nutricional modificam-se de acordo com o momento em que o paciente se encontra na trajetória de evolução da doença oncológica $^{19}$. As estratégias nutricionais deverão pautar-se, principalmente, no estado funcional e nutricional, na anamnese alimentar e nos aspectos bioéticos, como autonomia, tendo como meta proporcionar satisfação e conforto, visando primordialmente ao controle de sintomas e à melhora da qualidade de vida ${ }^{19,20}$.

Para realizar admissáo do paciente internado com suspeita ou confirmação de Covid-19, o nutricionista deve obter as informaçóes necessárias por meio dos familiares ou cuidadores do paciente, desde que náo contactantes diretos e que não apresentem sintomas de síndrome gripal, e por dados de prontuário e informaçōes fornecidas por membros da equipe interdisciplinar que já estejam em contato presencial com o paciente.

Deve-se evitar realizar avaliação nutricional presencial durante a assistência aos casos suspeitos ou confirmados de Covid-19 $9^{12,18}$. Entretanto, recomenda-se manter a avaliação nutricional dos pacientes, conforme protocolo institucional, sempre que possível. É preconizado o uso de dados clínicos, indicadores prognósticos, avaliaçáo de sintomas de impacto nutricional, parâmetros laboratoriais e instrumentos que possam ser preenchidos pelo paciente sem necessidade de auxílio ou pelo familiar, ou cuidador ${ }^{18,19}$.

A avaliação da ingestáo alimentar e o monitoramento da presença de sintomas devem ocorrer diariamente. Recomenda-se que o nutricionista utilize recursos de teleconsulta, dados registrados em prontuários e discussóes com a equipe interdisciplinar para orientar o planejamento do cuidado nutricional ${ }^{12}$.

As adequaçóes no plano alimentar e nutricional devem ser realizadas de acordo com avaliaçáo clínica, nutricional e conforme a sintomatologia apresentada, visando à promoção da adequada aceitação alimentar, ao auxílio no controle dos sintomas e à manutenção ou recuperação do estado nutricional, entre outros ${ }^{18,19}$. Considerar a terapia nutricional oral e enteral nos pacientes em risco nutricional e que não estejam na fase final de vida ${ }^{19}$, conforme protocolos institucionais.

$\mathrm{Na}$ alta hospitalar, o nutricionista deve realizar o aconselhamento nutricional ao paciente, quando possível; além de orientar seu familiar ou cuidador. Todas as orientaçóes nutricionais deverão ser fornecidas por escrito.

Os atendimentos realizados, as orientações fornecidas e o plano de cuidados nutricional devem ser registrados em prontuário ${ }^{19}$ e compartilhados com a equipe, de acordo com a rotina institucional.

Os alimentos e as bebidas ofertados aos pacientes internados devem ser produzidos atendendo às boas práticas de fabricação e de manipulaçáo de alimentos, de forma a garantir a entrega de preparaçóes seguras ${ }^{21}$. Os pacientes náo devem compartilhar pratos, copos, talheres e outros itens ${ }^{13}$, sendo recomendado uso de material descartável para acondicioná-los.

\section{PESQUISA CLÍNICA}

Entre as medidas para redução da circulação e aglomeração de pessoas nas unidades de saúde, recomenda-se a suspensão/adiamento das coletas de dados de pesquisas clínicas que já se encontravam em andamento, quando previstas entrevistas presenciais, grupos focais ou avaliaçóes físicas dos pacientes e profissionais, que podem repercutir no aumento da exposiçấo de indivíduos de maneira desnecessária. Deve-se priorizar a modalidade de trabalho remoto para os alunos vinculados aos programas de pesquisas.

Coletas de dados de pesquisas, de caráter retrospectivo, cujas informaçóes de interesse possam ser coletadas remotamente por meio do acesso às plataformas digitais, à consulta a prontuários eletrônicos e a outros programas institucionais existentes, poderão ser incentivadas.

Além disso, devem ser estimulados o desenvolvimento e a continuidade de pesquisas cuja metodologia preveja coleta de dados por meio de ligação telefônica, por via digital, ou por bancos ou bases de dados secundárias, e o desenvolvimento de trabalhos de revisão sistemática da literatura.

Em virtude da escassez de estudos na área específica de nutrição e câncer avançado associado à Covid-19, é essencial o fomento em pesquisas científicas nesse campo, com a finalidade de produzir evidências para um melhor cuidado desse grupo de indivíduos no contexto da pandemia.

\section{CONCLUSÃO}

Diante do exposto, a definição de novos fluxos e recomendaçóes com o objetivo da manutenção da 
qualidade do atendimento nutricional aos pacientes com câncer avançado durante a pandemia da Covid-19 é imprescindível. No entanto, sem se distanciar da abordagem para o controle de sintomas, conforto e promoçáo da qualidade de vida e de morte dos pacientes e de seus familiares/cuidadores, pautada na comunicação e no trabalho interdisciplinar. Sendo assim, busca-se, por meio desta publicação, auxiliar outros serviços de saúde a ofertarem um cuidado nutricional digno e de qualidade a pacientes com câncer avançado em cuidados paliativos acometidos pela Covid-19.

\section{CONTRIBUIÇÕES}

Karla Santos da Costa Rosa e Livia Costa de Oliveira contribuíram na concepçáo e delineamento do estudo, na redação, análise e interpretação do manuscrito. Emanuelly Varea Maria Wiegert, Rosane de Souza Santos e Mariana Fernandes Costa contribuíram igualmente na redação, análise e interpretação do manuscrito. Todas as autoras participaram da revisão crítica do manuscrito e aprovaram a versão final.

\section{DECLARAÇÃO DE CONFLITO DE INTERESSES}

Nada a declarar.

\section{FONTES DE FINANCIAMENTO}

Não há.

\section{REFERÊNCIAS}

1. World Health Organization [Internet]. Geneva: WHO; c2020. WHO Director-General's opening remarks at the media briefing on COVID-19; 2020 Mar 11 [cited 2020 May 18]. Available from: https://www.who.int/dg/ speeches/detail/who-director-general-s-opening-remarksat-the-media-briefing-on-covid-19---11-may-2020

2. Tay MZ, Poh CM, Rénia L, et al. The trinity of COVID-19: immunity, inflammation and intervention. Nat Rev Immunol. 2020 Apr 28. doi: https://doi. org/10.1038/s41577-020-0311-8 Epub ahead of print. PubMed PMID: 32346093.

3. Ministério da Saúde (BR). Diretrizes para diagnóstico e tratamento da COVID-19 [Internet]. Versão 3. Brasília, DF: Ministério da saúde; 2020 abr. 17 [acesso 2020 maio 12]. Disponível em: https://portalarquivos.saude.gov.br/ images/pdf/2020/April/18/Diretrizes-Covid19.pdf

4. Organização Pan-Americana da Saúde (BR) [Internet]. Brasília, DF: OPAS; [data desconhecida]. Folha informativa: COVID 19 (doença causada pelo novo Coronavírus); 2020 [acesso 2020 maio 12]. Disponível em: https://www.paho.org/bra/index.php?option=com_ content $\&$ view $=$ article\&id $=6101$ : covid $19 \&$ Itemid $=875$

5. Thuler LCS, Melo AC. Sars-CoV-2/Covid-19 em pacientes com câncer. Rev Bras Cancerol. 2020;66(2):e00970. doi: https://doi.org/10.32635/2176-9745. RBC.2020v66n2.970

6. Zhang L, Zhu F, Xie L, et. al. Clinical characteristics of COVID-19-infected cancer patients: a retrospective case study in three hospitals within Wuhan, China. Ann Oncol. 2020 Mar 26;S0923-7534(20)36383-3. doi: https://doi.org/10.1016/j.annonc.2020.03.296 Epub ahead of print. PubMed PMID: 32224151.

7. Al-Shamsi HO, Alhazzani W, Alhuraiji H, et al. A practical approach to the management of cancer patients during the novel coronavirus disease 2019 (COVID-19) Pandemic: an international collaborative group. Oncologist. 2020 Apr 3;25:1-10. doi: https:// doi.org/10.1634/theoncologist.2020-0213 Epub ahead of print. PubMed PMID: 32243668.

8. Singh AG, Deodhar J, Chatuverdi P. Navigating the impact of COVID-19 on palliative care for head and neck cancer. Head Neck. 2020;1-3. doi: https://doi. org/10.1002/hed.26211

9. International Association for Hospice and Palliative Care [Internet]. Houston: IAHPC; [data desconhecida]. Global palliative care organizations; [cited 2020 May 18]. Available from: http:/globalpalliativecare.org/covid-19/

10. World Health Organization. Palliative Care. Geneva: WHO; 2007. (Cancer control: knowledge into action: WHO guide for effective programs; module 05).

11. Barazzoni R, Bischoff SC, Breda J, et al. ESPEN expert statements and practical guidance for nutritional management of individuals with SARS-CoV-2 infection. Clin Nutr. 2020 Mar 31. doi: https://doi.org/10.1016/j. clnu.2020.03.022 Epub ahead of print. PubMed PMID: 32305181 .

12. Piovarcari SMF, Santos GFCG, Santana GA, et al. Fluxo de assistência nutricional para pacientes admitidos com COVID-19 e SCOVID-19 em unidade hospitalar. BRASPEN J [Internet]. 2020 [acesso 2020 maio 18];35(1):6-8. Disponível em: https://66b28c71-9a364ddb-9739-12f146d519be.usrfiles.com/ugd/66b28c_2 f5d298499184d22b2655dff908f58c9.pdf.

13. Agência Nacional de Vigilância Sanitária. Nota Técnica GVIMS/GGTES/ANVISA No 04 2020: orientaçôes para serviços de saúde: medidas de prevenção e controle que devem ser adotadas durante a assistência aos casos suspeitos ou confirmados de infecçáo pelo novo coronavírus (SARS-CoV-2) [Internet]. Brasília, DF: ANVISA; 2020 jan 30 [atualizada 2020 maio 08; acesso 2020 maio 15]. Disponível em: http:// portal.anvisa.gov.br/documents/33852/271858/ Nota+T\%C3\%A9cnica+n+04-2020+GVIMS-GGTESANVISA/ab598660-3de4-4f14-8e6f-b9341c196b28 
14. Academia Nacional de Cuidados Paliativos. Posicionamento da Academia Nacional de cuidados paliativos sobre COVID-19 [Internet]. São Paulo: ANCP; 2020. [acesso 2020 maio 14]. Disponível em: https://www.dropbox.com/s/g77qi6xk2jc8rky/FINAL ANCP_Ebook_cuidados_COVID-19.pdf?dl=0

15. World Health Organization. Home care for patients with suspected novel coronavirus $(\mathrm{nCoV})$ infection presenting with mild symptoms and management of contacts: interim guidance. Geneva: WHO; 2020 Jan 20.

16. Conselho Federal de Nutricionistas (BR). Resolução CFN no 646, de 18 de março de 2020. Suspende até o dia 31 de agosto de 2020 o disposto no artigo 36 da Resoluçáo CFN $\mathrm{n}^{\circ}$ 599, de 25 de fevereiro de 2018, que aprova o Código de Ética e de Conduta dos Nutricionistas [Internet]. Diário Oficial da União, Brasília, DF; 2020 mar 19. Seção I, p. 81 [acesso 2020 maio 10]. Disponível em: https://www. cfn.org.br/wp-content/uploads/2020/03/Resol-CFN646-codigo-etica.pdf

17. Instituto Nacional de Câncer José Alencar Gomes da Silva. Hospital do Câncer IV: planejamento emergencial para enfrentamento diante da pandemia causada pelo SARS-CoV-2 (COVID -19). Rio de Janeiro: INCA; $2020 \mathrm{abr}$.

18. Nabarrete JM, D’Almeida CA, Castro MG, et al. Recomendaçóes nutricionais para o paciente com câncer durante a pandemia do coronavírus (COVID-19). BRASPEN J. 2020;35(1):9-12. doi: https://doi. org/10.37111/braspenj.AE2020351002

19. Instituto Nacional de Câncer José Alencar Gomes da Silva. Consenso nacional de nutrição oncológica. 2 ed. rev. ampl. atual. Rio de Janeiro: INCA; 2015. Capítulo IV, Assistência nutricional ao paciente oncológico adulto em cuidados paliativos; p. 61.

20. Rosaneli CF, organizadora. Contexto, conflitos e escolhas em alimentação e bioética. Curitiba: PUCPRess; 2016.

21. Agência Nacional de Vigilância Sanitária (BR). Resolução RDC no 216, de 15 de setembro de 2004. Dispóe sobre Regulamento Técnico de Boas Práticas para Serviços de Alimentação. Diário Oficial da União, Brasília, DF; 2004 set 16. 